\title{
A cardiology fellow's take-home points from ASNC's Webinar: Cases in Tc 99m-PYP evaluation of ATTR cardiac amyloidosis - interpretation and reporting
}

\author{
Mrinali Shetty, $M^{\mathrm{a}}$ \\ a University of Chicago (NorthShore), NorthShore University Health System, Evanston, IL
}

Received Oct 19, 2020; accepted Dec 14, 2020

doi: $10.1007 / \mathrm{s} 12350-020-02516-3$

Cardiac amyloidosis (CA) is a rapidly progressing cardiomyopathy which has garnered substantial attention due to recent major advancements in diagnostic testing and treatment modalities for a disease that was once considered incurable. ${ }^{1}$ If untreated, median survival from diagnosis is $<6$ months for light chain cardiac amyloidosis (AL-CA) and 3 to 5 years for transthyretin cardiac amyloidosis (TTR-CA). ${ }^{2,3}$ Technetium-99m pyrophosphate (Tc-99m PYP) scintigraphy has a high diagnostic accuracy for TTR-CA and can be performed by any laboratory with access to a SPECT camera and Tc-99m PYP or another bone tracer. The high diagnostic accuracy of Tc-99m PYP, along with the absence of paraproteinemia, is very specific for TTR$\mathrm{CA}$, obviating the need for endomyocardial biopsies in most patients with suspected TTR-CA.

With the rapid evolution in imaging-based diagnosis of cardiac amyloidosis and the expected increase in the performance of clinically indicated Tc-99m PYP studies, there is an ongoing need for focused education. A recent ASNC webinar "Cases in 99m-Tc-PYP Evaluation of ATTR Cardiac Amyloidosis: Interpretation and Reporting" provided in-depth discussion on the evaluation of Tc-99m PYP studies. This webinar featured real-life cases from several high-volume centers in the country and highlighted the imaging and interpretive challenges associated with contemporaneous Tc-99m PYP scintigraphy. Like most cardiology fellows, the

All editorial decisions for this article, including selection of reviewers and the final decision, were made by guest editor Saurabh Malhotra, $\mathrm{MD}, \mathrm{MPH}$.

Reprint requests: Mrinali Shetty, MD, University of Chicago (NorthShore), NorthShore University Health System, 2650 Ridge Ave, Suite 1304, Evanston, IL 60201; mrinali.shetty@uchospitals.edu J Nucl Cardiol 2021;28:604-9.

$1071-3581 / \$ 34.00$

Copyright (c) 2021 American Society of Nuclear Cardiology.
COVID-19 pandemic had affected my training due to the precipitous drop in cardiac diagnostic testing and interventions at the height of the pandemic. Similar to other live online educational initiatives led by ASNC, I found this focused webinar to be highly instructive and practical (Figure 1). Herein, I summarize the salient attributes of this webinar, with the aim of educating other FITs and cardiologists alike, on the interpretation and reporting of Tc-99m scintigraphy.

\section{RATIONALE FOR TC-99M PYP SCINTIGRAPHY}

Myocardial uptake of Tc-99m PYP, a bone-avid radiotracer, has a high diagnostic accuracy for TTR-CA. When appropriate technique and recognition of potential confounders are accounted for, the diagnostic accuracy of Tc-99m PYP scans for TTR-CA is extremely high, with a reported sensitivity of $>99 \%$. A visual grade of 2 or 3 uptake on planar images, combined with the absence of a monoclonal protein spike in serum or urine, has a reported specificity of $100 \% .^{4}$ Tc-99m PYP scintigraphy is used in conjunction with echocardiography or cardiac magnetic resonance for the diagnosis of TTR-CA, and the latter modalities provide integral structural and hemodynamic assessment. ${ }^{5}$

\section{INTERPRETATION OF TC-99M PYP SCINTIGRAPHY}

Careful evaluation of PYP images in a stepwise fashion is required.

Step 1 Tomographic reconstruction

Tomographic acquisition of Tc-99m PYP is highly recommended to confirm myocardial PYP uptake.

(a) Assess radiotracer uptake in the myocardium on SPECT imaging 


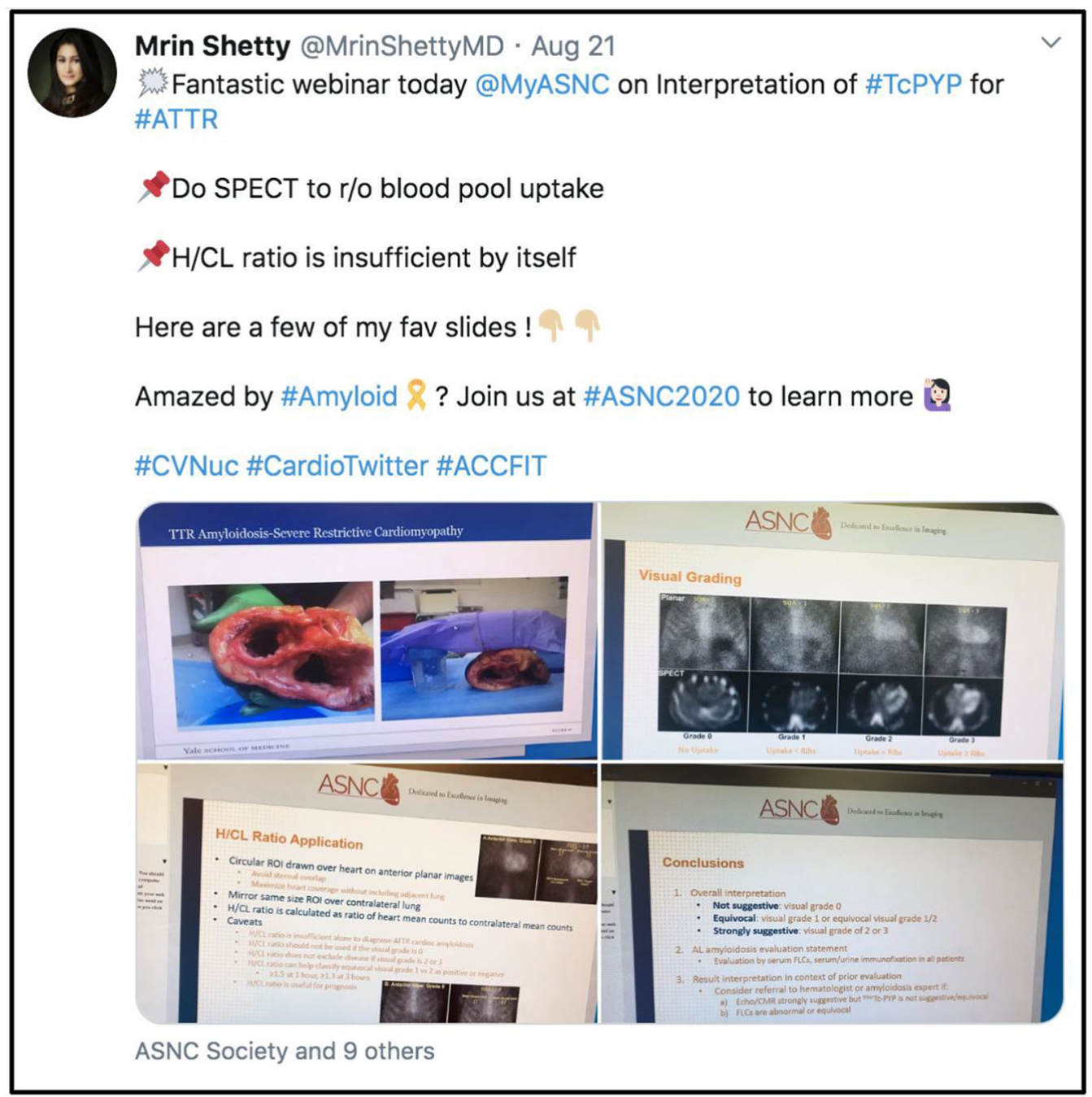

Figure 1. \#CardioTwitter is an invaluable resource to stay up to date with the latest in the Science and connect with thought leaders. Here is a tweet of my favorite slides from the talk. Follow hashtags like \#CVNuc and \#ASNC2020 for tweets pertaining to nuclear cardiology.

- If no uptake in the myocardium $\rightarrow$ the study is negative

- If uptake is present $\rightarrow$ proceed to 'Step 2,' i.e., visual grading

(b) Differentiate myocardial uptake from the blood pool

- Repeat imaging at 3 hours if excessive blood pool activity is noted at 1 hour.

Step 2 Semi-quantitative visual grading - comparison of myocardial uptake to rib uptake

Semi-quantitative visual grading of radiolabeled tracer uptake in the myocardium was first reported by Perugini et. al using 99mTc-3,3-diphosphono-1,2- propanodicarboxylic acid (Tc-99m DPD) in a cohort of mixed TTR and AL cardiac amyloidosis. Sensitivity and specificity for TTR-CA were $100 \%$ using $99 \mathrm{~m}$ Tc DPD. ${ }^{6}$ This grading scale has come to be known as the 'Perugini grading scale' (Figure 2).

Step 3 Heart-to-contralateral ratio assessment $(\mathrm{H} /$ CL ratio)

(a) Region of Interest (ROI) (Figures 3, 4).

- Heart ROI: In the anterior planar images, draw a circular ROI over the heart.

The ROI should maximize heart coverage without including adjacent lung or sternum. 

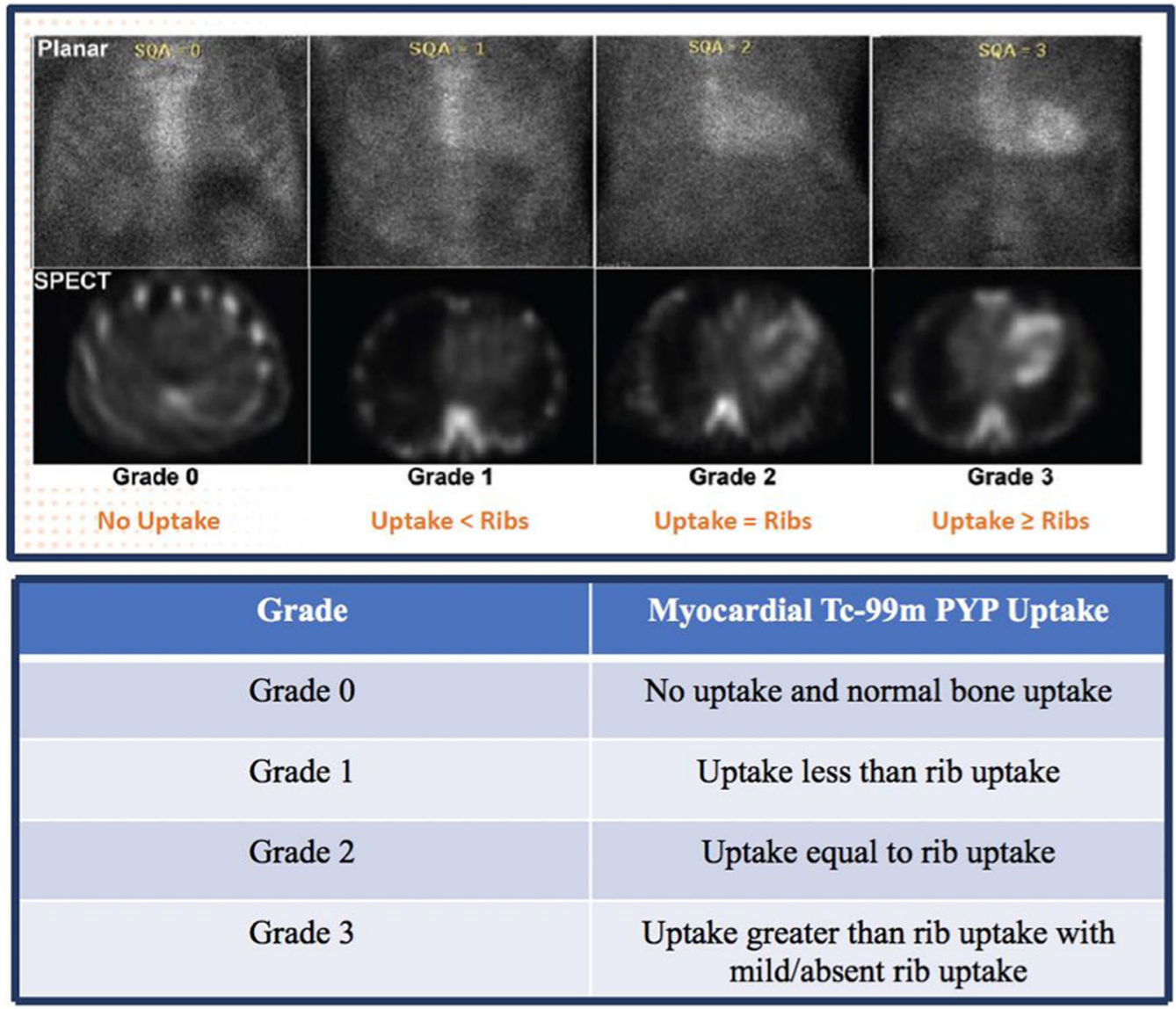

Figure 2. Semi-quantitative visual grading - comparison of myocardial uptake to rib uptake. [Image credit: James Bourque, MD MHS, UVA Health].

- Mirror the same size ROI over the contralateral chest

- $\mathrm{H} / \mathrm{CL}$ ratio is the ratio of heart mean counts to contralateral lung mean counts

(b) Caveats:

- $\mathrm{H} / \mathrm{CL}$ ratio is useful for prognosis, but it is insufficient for diagnosis of TTR-CA

- Cannot be used if visual grade is 0

- Can be used to classify equivocal studies (grade 1 vs 2) as positive or negative based on the ratio: Positive $\rightarrow$ ratio of $\geq 1.5$ at 1 hour, $\geq 1.3$ at 3 hours

- Does not exclude disease if visual grade is 2 or 3

ASNC consensus recommendations for PYP scintigraphy consider a scan to be positive if the visual grade is $\geq 2$ while also highlighting the high diagnostic accuracy of an H/CL ratio $\geq 1.5$ at 1 hour. $^{7}$ Sources of error on PYP scintigraphy are listed in Table 1.

\section{REPORTING OF TC-99M PYP SCINTIGRAPHY}

The ASNC guideline for myocardial Tc-99m PYP imaging has defined certain parameters which must be incorporated in a report. This includes 5 key elements:

1. Demographics indication for the test, prior imaging, biopsy results if performed

2. Method radiotracer dose, interval between injection and scan, scan technique (planar and SPECT)

3. Findings

- Image quality

- Visual scan interpretation

- Semi-quantitative interpretation

- $\mathrm{H} / \mathrm{CL}$ ratio (recommended for positive scans)

4. Ancillary findings whole body imaging, CT attenuation correction

5. Conclusion:

- Overall interpretation

Not suggestive: Visual grade 0 

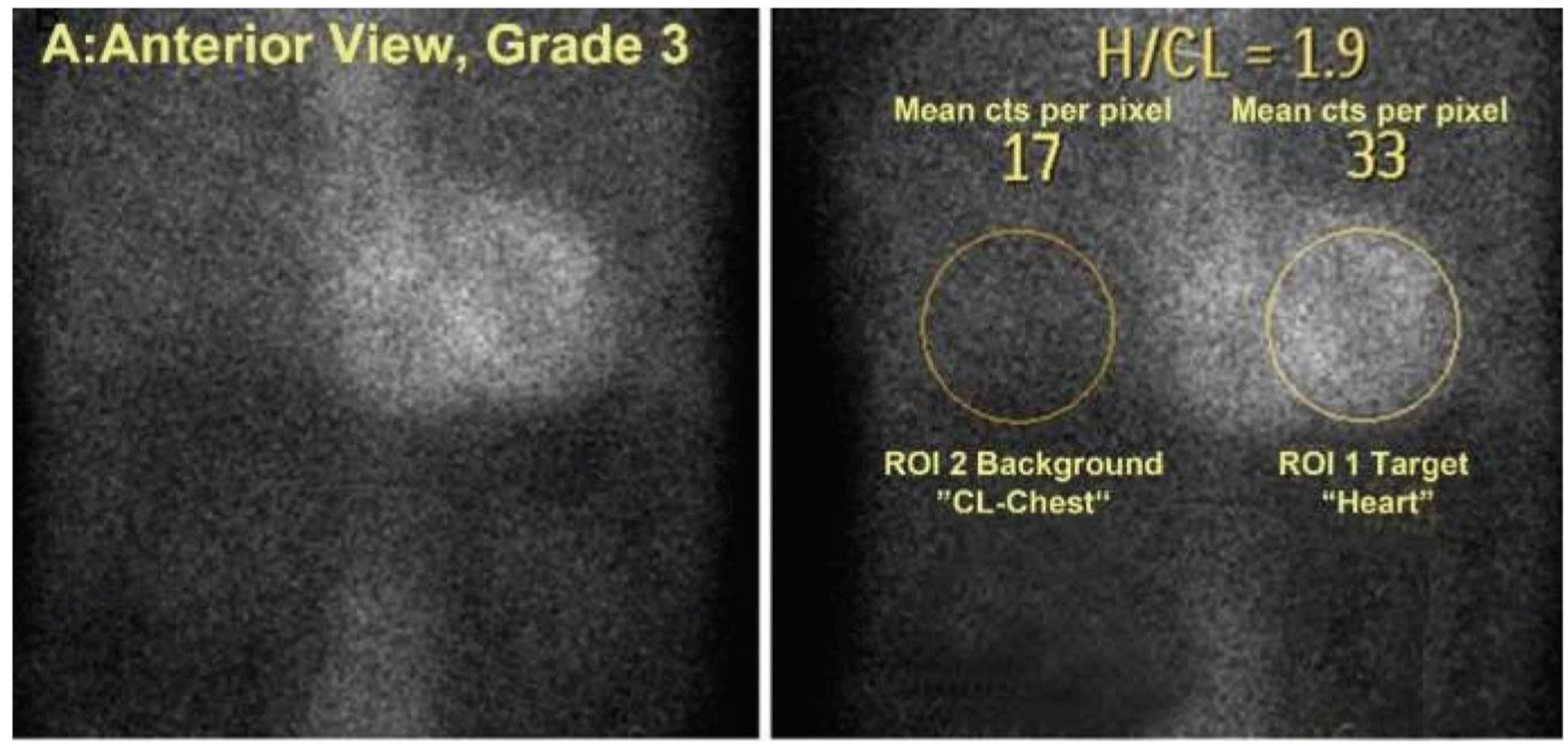

Figure 3. Methodology for assessment of heart-to-contralateral (H/CL) ratio. ROI 1 encircles the heart and has 33 mean counts per pixel. ROI 2 encircles the contralateral chest and has 17 mean counts per pixel. Therefore, the H/CL ratio is 1.9. [Image credit: James Bourque, MD MHS, UVA Health].
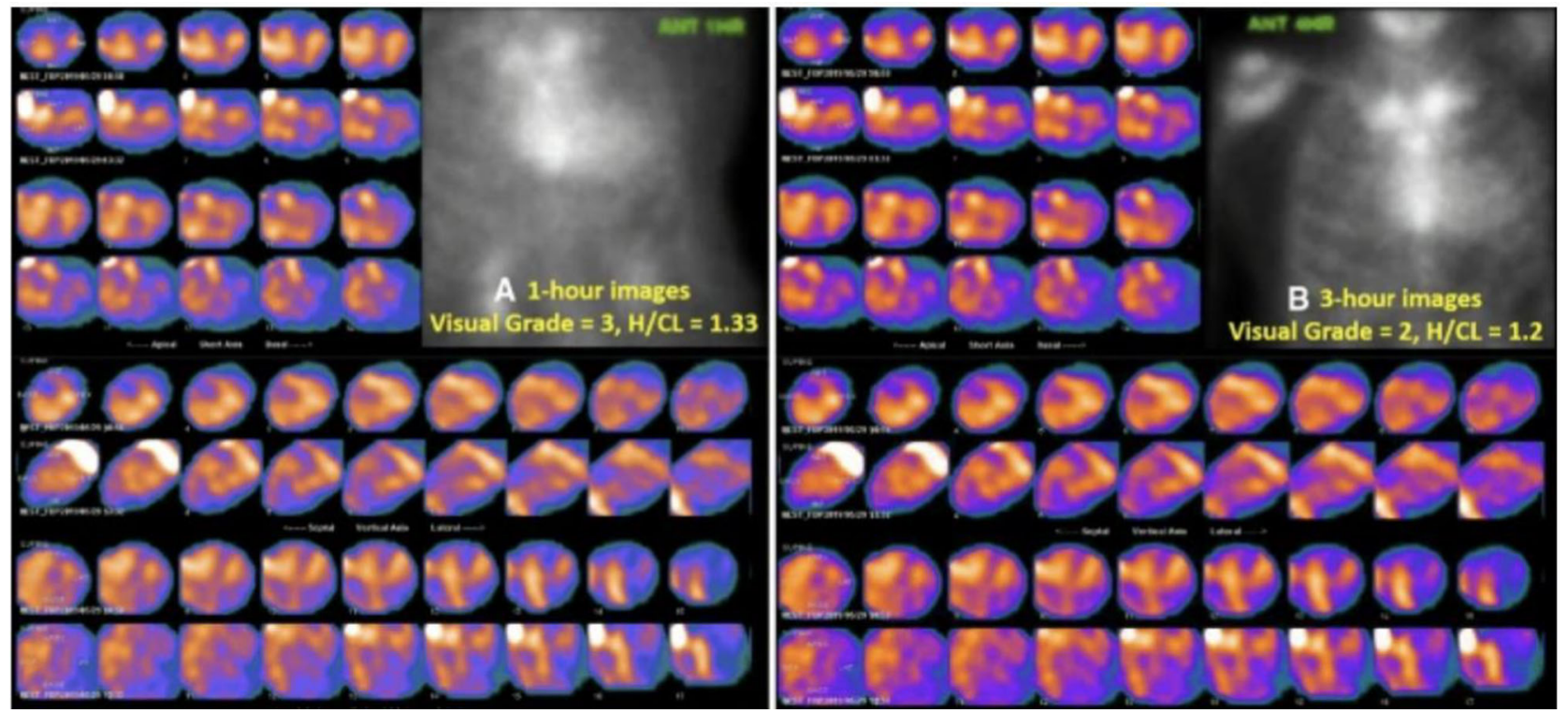

Figure 4. Persistence of blood pool activity can be seen at 3 hours in patients with severely reduced left ventricular function. PYP SPECT aides in identifying no myocardial isotope uptake when activity was noted on A 1-hour and B 3-hour planar PYP imaging. Thus given the diagnostic, therapeutic, and prognostic implications, planar findings should be confirmed with PYP SPECT imaging. [Image credit: ${ }^{19}$ ]. 
Table 1. Sources of error on PYP scintigraphy ${ }^{16-19}$

\section{Sources of error on PYP scintigraphy}

$\mathrm{H} / \mathrm{CL}$ Ratio Since the $\mathrm{H} / \mathrm{CL}$ ratio relies on extra-cardiac tissue for comparison, pathology affecting these sites can lead to erroneous results. Increased rib (metastasis) or muscle uptake, pleural or pericardial effusions may all skew the counts and thereby the ratio

Increased cardiac counts may be seen in mitral annular and aortic valve calcification..

Acute and subacute myocardial infarction may cause abnormal focal uptake.

The $\mathrm{H} / \mathrm{CL}$ ratio may be falsely low in patients with large myocardial scars as there is no uptake in these regions.

Visual Persistent blood pool activity at 1 hour may be seen in low cardiac output states and in patients with grade renal failure. In such cases, delayed planar imaging at 3 hours is recommended.

This brings up the important consideration of the addition of SPECT tomographic reconstruction to planar imaging due to its ability to differentiate myocardial uptake from blood pool activity. Planar imaging alone may result in studies being classified as indeterminate (Sperry and Asif papers). A majority of these stem from the $\mathrm{H} / \mathrm{CL}$ ratio, however, on occasion, the visual grade alone may misclassify a patient.

Given the diagnostic, therapeutic, and prognostic implications, planar findings should be confirmed with PYP SPECT imaging.

SPECT While not specifically reported, there is a theoretical possibility that focal PYP uptake may suggest that early TTR-CA could be missed on SPECT.

Blood pool activity could mask early, focal uptake.

Hybrid imaging with SPECT/CT could improve diagnostic accuracy in such cases.

$1 / 2$

Equivocal: Visual grade 1 or equivocal visual grade

Strongly suggestive: visual grade 2 or 3

- Highlight that a negative or mildly positive scan does not exclude AL- CA and that equivocal results could represent either AL-CA or early TTR-CA

- Recommend performance of - serum-free light chains, serum and urine immunofixation to rule out AL-CA.

- Consider referral to an amyloidosis expert or hematologist if the echo or CMR is strongly suggestive but the Tc-99m PYP study is not suggestive/equivocal or if the free light chains are abnormal or equivocal.

\section{CRUCIAL CONCEPTS}

(1) TTR-CA is not a rare disease. Observational studies suggest TTR-CA to be prevalent in $13 \%$ of geriatric patients admitted with heart failure and reduced ejection fraction in $16 \%$ of patients undergoing transcatheter aortic valve replacement for aortic stenosis and in $10 \%$ of patients with bilateral carpal tunnel syndrome. ${ }^{8-11}$

(2) The availability of targeted therapies for TTR-CA has put an emphasis on early accurate diagnosis. $^{12-14}$ Medical therapy for TTTR-CA is more effective when initiated early-on in the disease process. $^{15}$

(3) Tc-99m PYP imaging with its high diagnostic accuracy has negated the need for invasive endomyocardial biopsies in the majority of cases of ATTRCA to confirm diagnosis.

(4) It is felicitous for cardiology fellows-in-training to acquire in-depth understanding of the indications, advantages, and pitfalls of Tc-99m PYP scintigraphy. Effective imaging is vital for the diagnosis, prognosis, and therapeutic management for patients with TTR-CA.

\section{References}

1. Singh V, Falk R, Di Carli MF, Kijewski M, Rapezzi C, Dorbala S (2019) State-of-the-art radionuclide imaging in cardiac transthyretin amyloidosis. J Nucl Cardiol 26:158-73

2. Falk RH, Alexander KM, Liao R, Dorbala S (2016) AL (LightChain) cardiac amyloidosis. J Am Coll Cardiol 68:1323

3. Ruberg Frederick L, Berk JL (2012) Transthyretin (TTR) cardiac amyloidosis. Circulation 126:1286-1300

4. Gillmore Julian D, Maurer Mathew S, Falk Rodney H et al (2016) Nonbiopsy diagnosis of cardiac transthyretin amyloidosis. Circulation 133:2404-12 
5. Dorbala S, Cuddy S, Falk RH (2020) How to image cardiac amyloidosis. JACC: Cardiovasc Imaging 13:1368

6. Perugini E, Guidalotti PL, Salvi F et al (2005) Noninvasive etiologic diagnosis of cardiac amyloidosis using $99 \mathrm{mTc}-3,3-$ diphosphono-1,2-propanodicarboxylic acid scintigraphy. J Am Coll Cardiol 46:1076-84

7. Dorbala S, Ando Y, Bokhari S et al (2019) ASNC/AHA/ASE/ EANM/HFSA/ISA/SCMR/SNMMI expert consensus recommendations for multimodality imaging in cardiac amyloidosis: Part 1 of 2-evidence base and standardized methods of imaging. J Cardiac Fail 25:e1-e39

8. González-López E, Gallego-Delgado M, Guzzo-Merello G et al (2015) Wild-type transthyretin amyloidosis as a cause of heart failure with preserved ejection fraction. Eur Heart J 36:2585-94

9. Castaño A, Narotsky DL, Hamid N et al (2017) Unveiling transthyretin cardiac amyloidosis and its predictors among elderly patients with severe aortic stenosis undergoing transcatheter aortic valve replacement. Eur Heart J 38:2879-87

10. Sperry BW, Reyes BA, Ikram A et al (2018) Tenosynovial and cardiac amyloidosis in patients undergoing carpal tunnel release. J Am Coll Cardiol 72:2040-50

11. Fosbøl EL, Rørth R, Leicht BP et al (2019) Association of carpal tunnel syndrome with amyloidosis, heart failure, and adverse cardiovascular outcomes. J Am Coll Cardiol 74:15

12. Maurer MS, Schwartz JH, Gundapaneni B et al (2018) Tafamidis treatment for patients with transthyretin amyloid cardiomyopathy. N Engl J Med 379:1007-16
13. Adams D, Gonzalez-Duarte A, O'Riordan WD et al (2018) Patisiran, an RNAi therapeutic, for hereditary transthyretin amyloidosis. N Engl J Med 379:11-21

14. Benson MD, Waddington-Cruz M, Berk JL et al (2018) Inotersen treatment for patients with hereditary transthyretin amyloidosis. N Engl J Med 379:22-31

15. Bokhari S, Cerqueira MD (2020) Tc-99m-PYP imaging for cardiac amyloidosis: Defining the best protocol before the flood gates burst. J NuclCardiol 27:1816

16. Wehbe RM, Kansal P, Holly TA (2020) Cases from a busy nuclear cardiology laboratory: Potential pitfalls in the interpretation of cardiac scintigraphy for ATTR cardiac amyloidosis. J Nucl Cardiol. https://doi.org/10.1007/s12350-020-02094-4

17. Murray CSG, Moadel RM, Tauras JM, Zamora E, Travin MI (2020) A potential pitfall in the use of 99mTc-PYP imaging for diagnosing cardiac ATTR amyloidosis. J Nucl Cardiol. https://doi. org/10.1007/s12350-020-02199-w

18. Régis C, Harel F, Martineau P et al (2020) Tc-99m-pyrophosphate scintigraphy for the diagnosis of ATTR cardiac amyloidosis: Comparison of quantitative and semi-quantitative approaches. J Nucl Cardiol 27:1808

19. Asif T, Gomez J, Singh V, Doukky R, Nedeltcheva A, Malhotra S (2020) Comparison of planar with tomographic pyrophosphate scintigraphy for transthyretin cardiac amyloidosis: Perils and pitfalls. J Nucl Cardiol. https://doi.org/10.1007/s12350-020-02328-5

Publisher's Note Springer Nature remains neutral with regard to jurisdictional claims in published maps and institutional affiliations. 